\title{
Development of a 3D bio-printer using CoreXY mechanism and syringe-based extrusion
}

\author{
Chin Fhong Soon ${ }^{1}$, Mohammad Faez Ramilan ${ }^{2}$, Dirman Hanafi ${ }^{3}$, Wan Nurshazwani Wan Zakaria ${ }^{4}$, \\ Safinaz Binti Mohd Khialdin ${ }^{5}$, Hazlita Isa ${ }^{6}$, Kian Sek Tee ${ }^{7}$ \\ ${ }^{1}$ Biosensor and Bioengineering Laboratory, MiNT-SRC, Universiti Tun Hussein Onn Malaysia, Malaysia \\ ${ }^{2,3,4,7}$ Faculty of Electrical and Electronic Engineering, Universiti Tun Hussein Onn Malaysia, Malaysia \\ ${ }^{5,6}$ Department of Ophthalmology, Pusat Kesihatan University Kebangsaan Malaysia, Malaysia
}

\begin{tabular}{l} 
Article Info \\
\hline Article history: \\
Received Jan Oct 9, 2019 \\
Revised Nov 10, 2019 \\
Accepted Dec 24, 2019 \\
\hline Keywords: \\
3D Bio-printer \\
Core XY mechanism \\
Fused deposition modelling \\
(FDM) \\
Syringe-based extruder
\end{tabular}

\begin{abstract}
3D Bio-printer is said to have potential in the applications of medical studies and tissue engineering. A 3D Bio-printer could be used to print the artificial organs for organs transplant and cell tissue culture for generating new cell tissue. It could help in future medical and biological research. This paper presents the development process, i.e. working principle of Fused Deposition Modelling (FDM), mechanical, electrical and related applied software, of a 3D Bio-printer using syringe-based extruder and Core XY mechanism. Up to date, FDM is a popular technique because it is easily implemented, precise and accurate. The printed product revealed high repeativity, where (sample mean, sample standard deviation) is $(0.72,0.2455) \mathrm{mm}$. The procedure could help future customized 3D Bio-printer.
\end{abstract}

Copyright () 2020 Institute of Advanced Engineering and Science. All rights reserved.

\section{Corresponding Author:}

Chin Fhong Soon,

Biosensor and Bioengineering Laboratory,

MiNT-SRC, Universiti Tun Hussein Onn Malaysia, Malaysia.

Email: soon@uthm.edu.my

\section{INTRODUCTION}

The earliest 3D printing technique was first introduced by Charles "Chuck" Hull in 1986 in which the 3D printer solidify the ink using light radiation and print the 3D model [1]. There are types of 3D printers available in the market such as Stereolithigraphy (SLA), Digital Light Processing (DLP), Fused Deposition Modelling (FDM), Selective Laser Sintering (SLS), Selective Laser Melting (SLM) and etc. [2-6]. FDM-typed 3D printer is the most famous among all the 3D printers in which the printer build a 3D model layer by layer from bottom to upward [7]. The ink of the 3D-printer is molten layer of plastic that fused up together [7].

The 3D printer evolved rapidly in many fields nowadays and of the famous field is in medical. Lately, 3D Bio-printer became vital in medical fields in printing artificial organs, tissue, skins and bones [8-14]. Millions of patients are dying every year in the process of long term waiting for organ transplant. In medical treatment, there are difficulties in obtaining the healthy organs like the lack of organ donors, suitability of the organs on blood type, the condition of patients and the ages of the patients [15, 16]. 3D Bio-printer plays an important role to cover the shortage of healthy and suitable organ for those who may need them.

M. Ozturk et.al [17] described that there are three techniques of 3D Bio-printing commercially used today such as Inkjet-Based Printing, Extrusion Based Printing and Laser Based-Write. These facts are also support by the journal published by M. Calado et.al [18]. C. Ventola et.al [19] stated that 3D bio-printing are very famous especially in medical fields for research and study in many fields of researches such as tissue engineering, regenerative medicine and organ transplant since 2000s. The first 3D bio-printer [20] 
is introduced by Wake Forest Institute in year 1999 in which a fully functioned artificial urinary bladder was successfully printed. In year 2002, once again in medical field, 3D bio-printing produced artificial kidney that 100 percent replicated to the original one using 'bio-ink' technique. There are many other applications involving 3D Bio-printing such as Orthodontic which is one of the medical field that widely used of 3D Bioprinting in producing Invisalign Braces for repairing the teeth alignment [21]. The other application of 3D Bio-printing is on anatomical model and surgical preparation which give a big help in assisting surgery doctor [22-24]. Last but not least, 3D Bio-printing is applied in Pathology field, the 3D Bio-printing is used to print polypeptide model of the disease structure for diseases study purpose, type of disease and biological structure of diseases [25-26]. This paper intend to propose the development of 3D Bio-printer based on the Fused Deposition Modelling (FDM) using customized syringe based nozzle to print 3D Bio-model products.

\section{RESEARCH METHOD}

\subsection{System Overview}

The flow of the 3D Bio-printer system starts with creating a 3D model as the input of the system and was saved into a stereolithography (STL) file type. Then, a slicer program translates this model into individual layers. The slicer program named CURA was used to convert the model into g-codes which are transferred into a Secure Digital (SD) card. The g-codes list the instruction of functions and coordinates for the stepper motors to move synchronously. All these process were done in the computer. The SD card that contains g-code is inserted into the 3D Bio-printer controller. The 3D Bio-printer will execute and move according to the g-codes to print the desire 3D Bio-model. Figure 1 shows the overview on the 3D Bioprinter system.

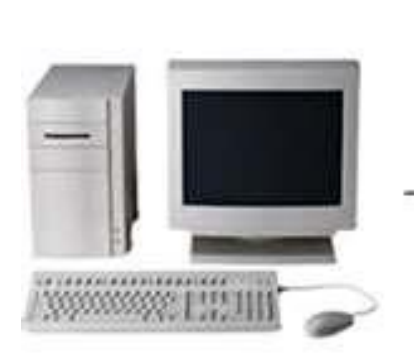

STL and gcode generation

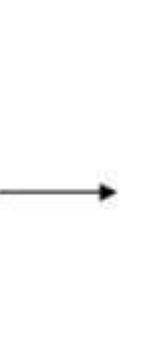

Transfer gcode and print

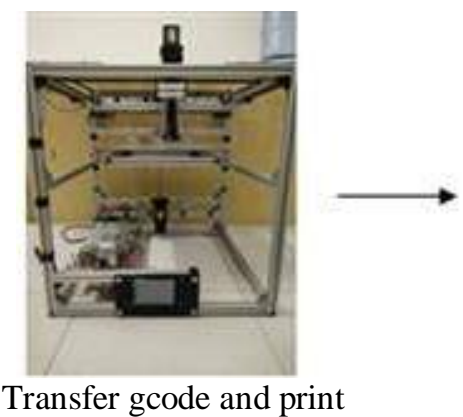

Figure 1. Overview on the 3D Bio-printer system

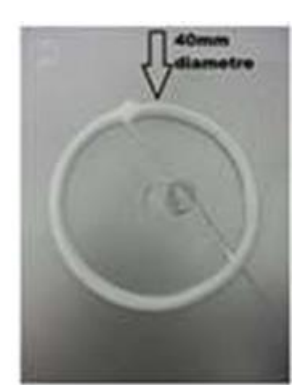

Printed product

\subsection{Mechanical Design}

The design of the 3D Bio-printer start with the software design where the SolidWorks was used to design the sketch for the customized 3D Bio-printer. SolidWorks is come in handy to sketch every component parts that are needed for building the 3D Bio-printer parts. The design of 3D Bio-printer includes the core XY mechanism in controlling movement of X and Y axis, Fused Deposition Model (FDM) printing technique and syringe-based extruder. Figure 2 shows the isometric view of the 3D Bio-printer. Its external dimensions are $(X, Y, Z)=(500 \mathrm{~mm}, 500 \mathrm{~mm}, 500 \mathrm{~mm})$.

Core $\mathrm{XY}$ mechanism is one of the mechanism for 3D bio-printer techniques that is widely used by the researchers. The Core XY mechanism was applied in this project for customized 3D Bio-printer. Figure 3 shows the sketch of Core XY mechanism where the belting connected the stepper motor of the X axis plane and $\mathrm{Y}$ axis plane for movement. A Core XY is a parallel manipulator system that positions XY coordinates of the nozzle in Cartesian plane. Limit switches are installed at end of $\mathrm{X}$ and $\mathrm{Y}$ axis.

The $\mathrm{Z}$ axis plane mechanism is upward and downward movement and is controlled by a stepper motor attached to a ball screw and supported by two sliders as shown in Figure 4. The origin, $(\mathrm{X}, \mathrm{Y}, \mathrm{Z})=$ $(0,0,0)$, is defined at lower left corner and upmost position.

The customized syringe-based extruder as shown in Figure 5, is used to extrude the gel-type ink. A single axis drive attached by a NEMA-17 stepper motor, is used to step and push the syringe plunger, hence squeezing gel-type ink in controlled volume. 


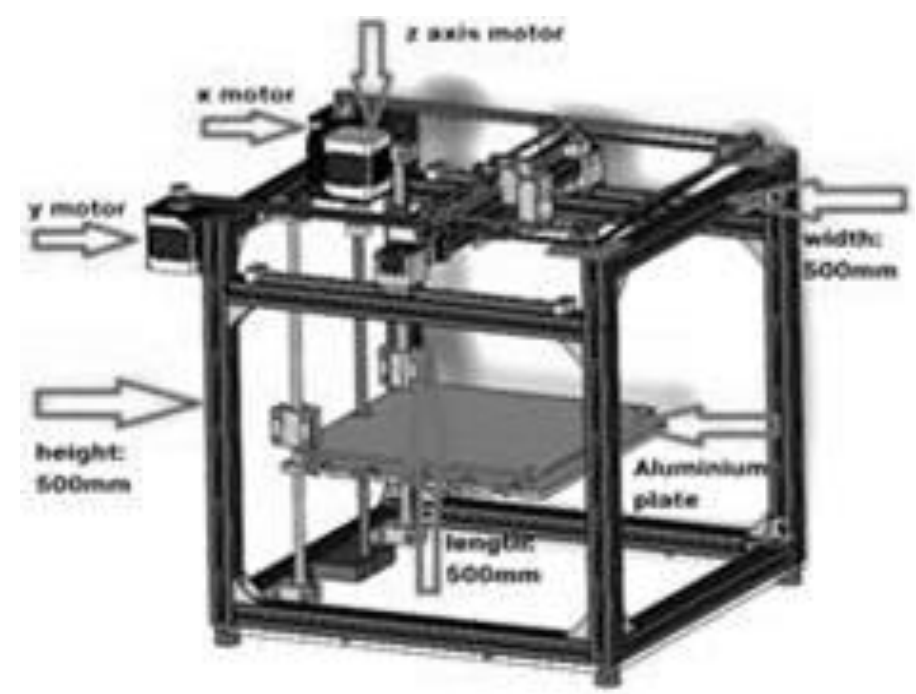

Figure 2. Isometric view of 3D Bio-printer

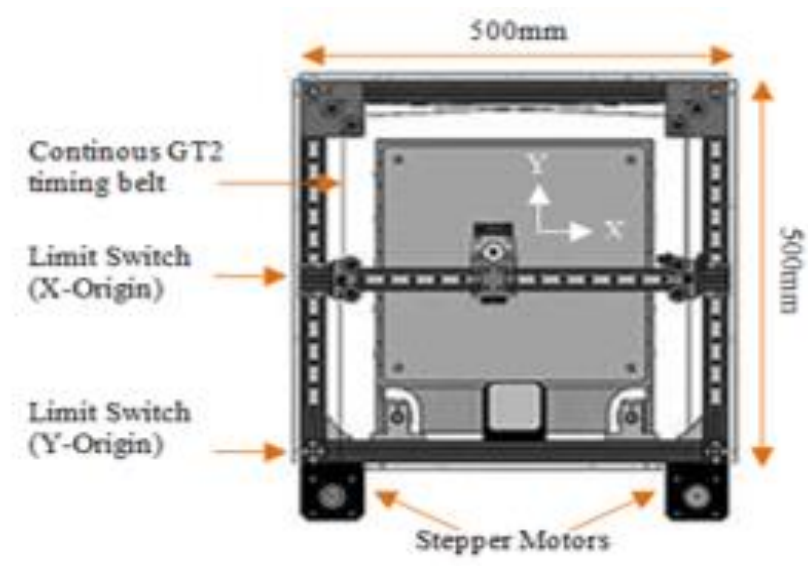

Figure 3. Core XY mechanism (Top view)

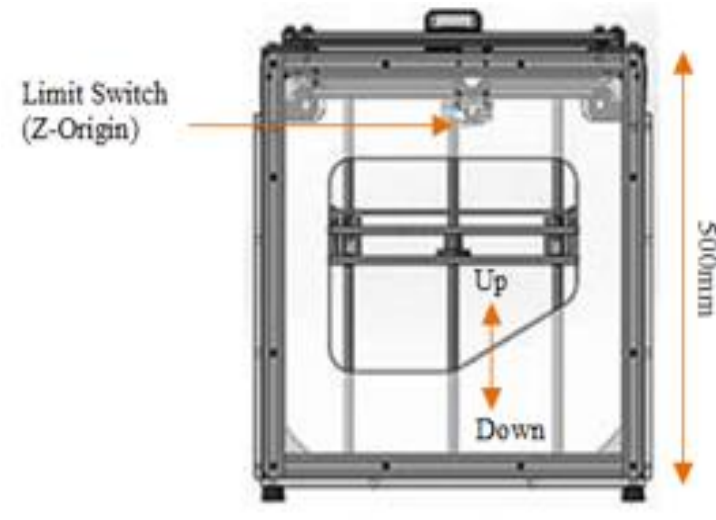

Figure 4. $\mathrm{Z}$ axis

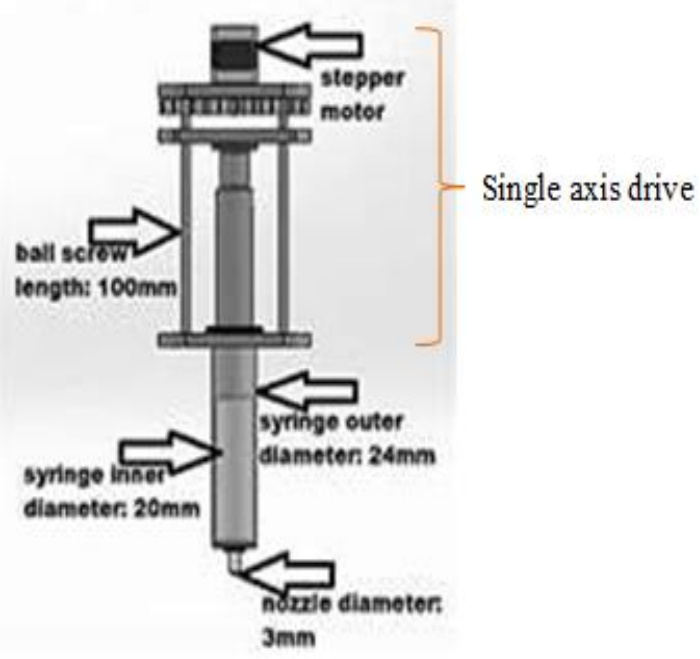

Figure 5. Syringe-based extruder 


\subsection{Electrical Design}

For electrical design, a 3D Controller named as CHITU (http://www.cbd-3d.com/en/index.shtml) is used as the controller for the 3D Bio-printer that reads and translates g-codes into corresponding NC movements of the Core XY, $\mathrm{Z}$ axis and Extruder in printing process. Figure 6 shows the schematic diagram for connection between mechanical components, electrical parts and the CHITU controller. The schematic diagram shows the connection for power source, stepper motors for each axis (X, Y, Z) and extruder, and limit switches that define the maximum and minimum strokes in each axis, i.e. aslo the origin, $[\mathrm{X}, \mathrm{Y}, \mathrm{Z}]=[0,0,0]$. The SD card slot is used to read from a SD memory card for the purposes of firmware flashing and printing. A 3.5" TFT colour touch screen provides an interface for monitoring and user instructions.

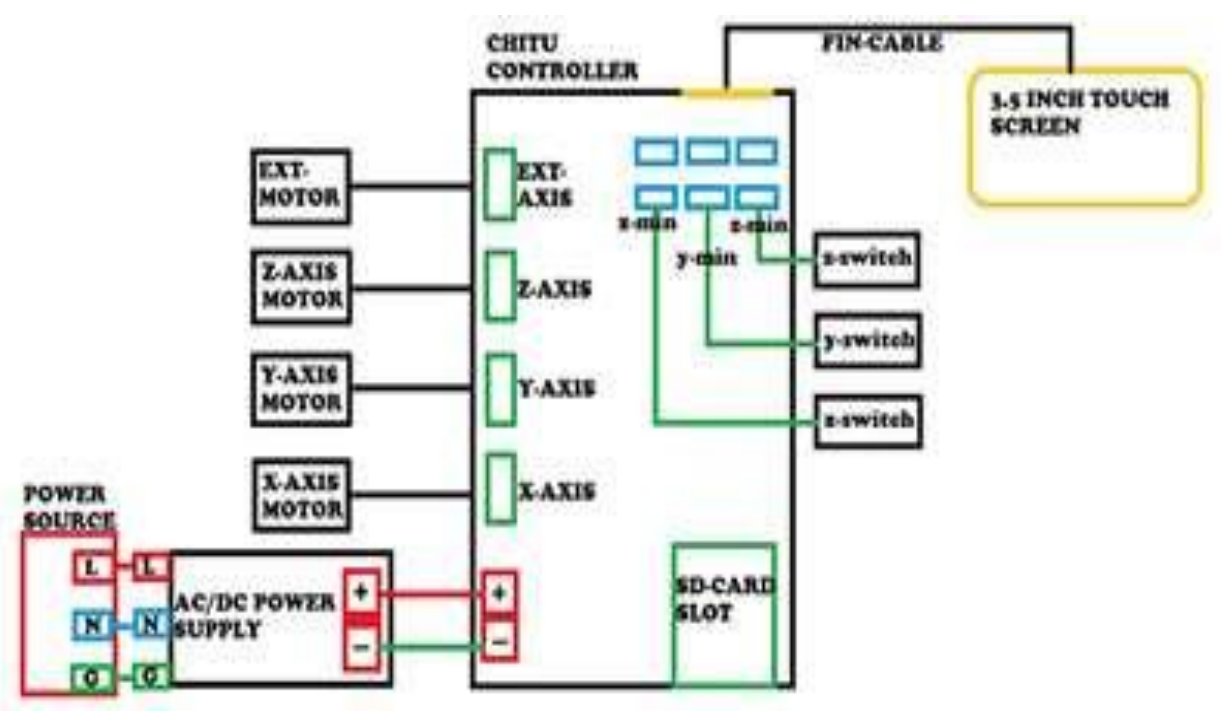

Figure 6. CHITU controller schematic diagram

\subsection{Calculation on Axis Movement}

Calculation for every single axis are vital to determine the suitable step size for each stepper motor. As shown in (1) is applied for the calculation. The step size are calculated and tabulated in Table 1.

$$
\text { Step Size }=\frac{\text { Distance per } \mathrm{rev} \times(\text { Angle } / \text { Step })}{16 \times \text { Step }}
$$

Table 1. Calculation on Step Size of X-axis, Y-axis, Z-axis and Extruder

\begin{tabular}{ccccc}
\hline Axis & Distance per revolution $(\mathrm{mm})$ & Angle $\left(^{\circ}\right)$ & Angle/Step $\left(^{\circ}\right)$ & Step Size $(\mathrm{mm} / \mathrm{step})$ \\
\hline $\mathrm{X}$ & $40.64^{*}$ & 360 & 1.8 & 0.0127000 \\
$\mathrm{Y}$ & $40.64^{*}$ & 360 & 1.8 & 0.0127000 \\
$\mathrm{Z}$ & $5^{* *}$ & 360 & 1.8 & 0.0015625 \\
Extruder & $5^{* *}$ & 360 & 1.8 & 0.0015625 \\
* Core XY $\mathbf{- 2 0}$ tooth GT2 timing pulley. Diameter $=\mathbf{2 . 0 3 2} \mathbf{m m} \times \mathbf{2 0}$ tooth $=\mathbf{4 0 . 6 4 m m}$ \\
** Ball screw pitch $=\mathbf{5 m m}$
\end{tabular}

\subsection{Calibration}

Calibration is the most important part of the research as it troubleshoots the problems occur while executing the 3D Bio-printer to reduce the error of the output. There are three types of calibration executed for $\mathrm{X}, \mathrm{Y}$ and $\mathrm{Z}$ axis movements such as manual calibration, exercised calibration and extruder calibration.

Manual calibration is the process that steps each axis manually and performs manual inspection using a ruler. Stepping length was entered and each axis stepped separately. Exercise program calibration is the calibration that involved with predefined g-codes with know movements. Several g-codes were coded to exercise three axis in performing point-to-point, linear paths, ramps and circles. The purpose of exercised g-code programs is to ensure the system works harmonically together. Extruder calibration is the calibration made for customized extruder only. The purpose of calibration is to check the movement of pushing the

Development of a 3D bio-printer using CoreXY mechanism and syringe-based extrusion (Chin Fhong Soon) 
plunger into the barrel of syringe. Manual adjustment of the speed and step size settings could be done to determine the extruded result.

\subsection{Hardware Assembly}

Figure 7 shows a completed customized 3D Bio-printer hardware with specification. All the main components are attached to the aluminium strut $20 \times 20 \mathrm{~mm}$ as the main frame of the 3D Bio-printer. Core XY and $\mathrm{Z}$ axis also are placed precisely inside the main frame. All electrical parts such as NEMA 17 Stepper motors and CHITU controller are wired accordingly.

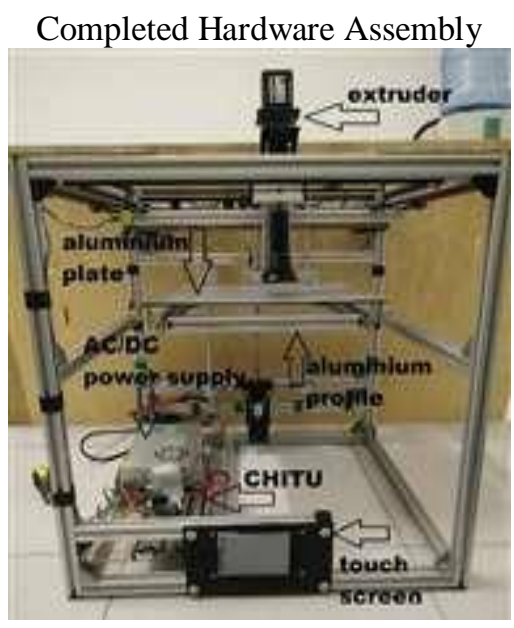

Specifications

Type: Fused Depositioning Modelling (Bio-Printing)

Dimension: 500 x 500 x $500 \mathrm{~mm} 3$

Weight: $12 \mathrm{~kg}$

Controller: CHITU Controller

Extruder: Syringe based

$\mathrm{X}$ and $\mathrm{Y}$ axis mechanism: Core $\mathrm{XY}$ type

Figure 7. Completed hardware assembly and specifications

\section{RESULTS AND ANALYSIS}

A 3D CAD design is needed before any 3D Bio-printing is executed. Figure 8 shows the full process on the CAD design of a ring-shaped 3D Bio-model. Firstly, the CAD drawing of the sample is designed by using SolidWorks with desired diameter value and shape as illustrated in Figure 8(a). Next, the CAD drawing file will be saved in STL file format as illustrated in Figure 8(b). Next, a STL file will be read by the Slicer software called CURA (https://ultimaker.com/en/products/ultimaker-cura-software) and the model is virtually sliced into multiple layers as illustrated in Figure 8(c). Every single layer contains streams of g-codes which will be executed by the 3D Bio-printer.

Table 2 shows the measured and error values for every single samples. The average measured value is $40.72 \mathrm{~mm}$ which is a difference of $0.72 \mathrm{~mm}$ to the target value. Meanwhile, the average error value is $0.72 \mathrm{~mm}$ because the printed product is collapsed due to the viscosity of toothpaste ink. The standard deviation for measured and error values is 0.2455 . One of the factor for this error could be the relative flow rate by nozzle size. The nozzle size has a diameter of $3 \mathrm{~mm}$ which might not be suitable for the usual flow rate setting. The vibration occurred in printer itself when printing may contribute to the error in printing. The vibration might cause the toothpaste to collapse and spread horizontally.

Table 2. Samples Results (target value $=40 \mathrm{~mm}$ )

\begin{tabular}{ccc}
\hline No. of Samples & Measured Values $(\mathrm{mm})$ & $\begin{array}{c}\text { Error Values } \\
\text { (Measured-Target) }(\mathrm{mm})\end{array}$ \\
\hline 1 & 41.0 & 1.0 \\
2 & 40.5 & 0.5 \\
3 & 40.7 & 0.7 \\
4 & 40.8 & 0.8 \\
5 & 40.4 & 0.4 \\
6 & 40.6 & 0.6 \\
7 & 40.7 & 0.7 \\
8 & 40.5 & 0.5 \\
9 & 40.8 & 0.8 \\
10 & 41.2 & 1.2 \\
Average & 40.72 & 0.72 \\
Standard Deviation & 0.2455 & 0.2455 \\
\hline
\end{tabular}


In this experiment, ten samples of the ring-shaped 3D model with 40mm diameter (inner) as shown in Figure 9 were printed. Tootepaste is used as the gel type specimen since it is the cheapest and easily available. All the samples are measured and calculated for data analysis.

(a)

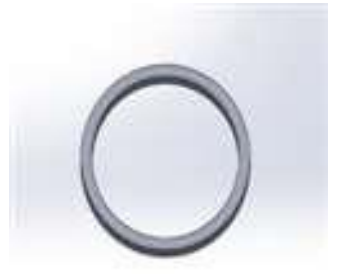

(b)

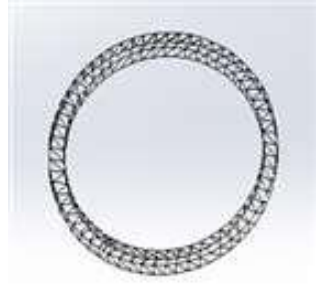

(c)

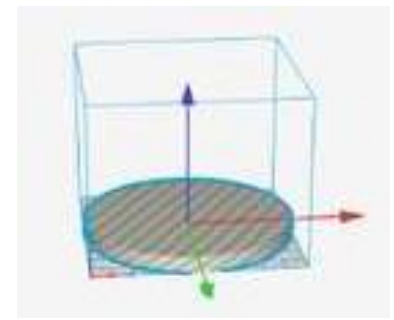

Figure 8. Full process on the CAD design of the ring-shaped 3D Bio-model; (a) Sample design in SolidWorks; (b) Sample design in STL format; (c) Sample design in CURA

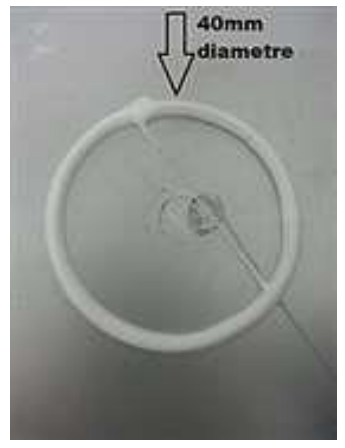

Figure 9. Printed product using toothpaste

\section{CONCLUSION}

The development of the customized 3D Bio-printer was a success in terms of software or hardware design. The customized 3D Bio-printer was able to function properly and print out the 3D Bio-model successfully according to CAD design with accepted error. This 3D Bio-printer can be further improved by implementing a more precise and accurate calculation on the alignment of the Core XY, by adding up the number of Limit switch used for more accurate positioning in Core XY and $\mathrm{Z}$ plane. The 3D Bio-printer can be further upgraded by using WiFi as communication system to transfer file from a computer terminal to a 3D Bio-printer wirelessly.

\section{ACKNOWLEDGEMENTS}

The research is financially supported by Fundamental Research Grant Scheme (FRGS) with Vot. No. K106 from the Ministry of Education Malaysia.

\section{REFERENCES}

[1] Hull, C.W., "Apparatus for production of three-dimensional objects by stereolithography." 1986, Google Patents.

[2] Mitteramskogler, G., et al., "Light curing strategies for lithography-based additive manufacturing of customized ceramics." Additive Manufacturing, 2014. 1: p. 110-118.

[3] Halloran, J.W., et al., "Photopolymerization of powder suspensions for shaping ceramics." Journal of the European Ceramic Society, 2011. 31(14): p. 2613-2619.

[4] Felzmann, R., et al., "Lithography-based additive manufacturing of cellular ceramic structures." Advanced Engineering Materials, 2012. 14(12): p. 1052-1058.

[5] Bellini, A., L. Shor, and S.I. Guceri, "New developments in fused deposition modeling of ceramics." Rapid Prototyping Journal, 2005. 11(4): p. 214-220.

[6] Wilkes, J., et al., "Additive manufacturing of $\mathrm{ZrO} 2-\mathrm{Al} 2 \mathrm{O} 3$ ceramic components by selective laser melting." Rapid Prototyping Journal, 2013. 19(1): p. 51-57. 
[7] Van Wijk, A. and I. van Wijk, “3D printing with biomaterials: Towards a sustainable and circular economy.” 2015: IOS press.

[8] Bechthold, L., et al., "3D printing: A qualitative assessment of applications, recent trends and the technology's future potential." 2015, Studien zum deutschen Innovationssystem.

[9] Ozbolat, I.T., W. Peng, and V. Ozbolat, “Application areas of 3D bioprinting.” Drug discovery today, 2016. 21(8): p. 1257-1271.

[10] Lee, V.K., et al., "Creating perfused functional vascular channels using 3D bio-printing technology." Biomaterials, 2014. 35(28): p. 8092-8102.

[11] Murphy, S.V. and A. Atala, "3D bioprinting of tissues and organs. Nature biotechnology,” 2014. 32(8): p. 773.

[12] Kolesky, D.B., et al., "3D bioprinting of vascularized, heterogeneous cell-laden tissue constructs." Advanced materials, 2014. 26(19): p. 3124-3130.

[13] Kang, H.-W., et al., "A 3D bioprinting system to produce human-scale tissue constructs with structural integrity." Nature biotechnology, 2016. 34(3): p. 312.

[14] Mandrycky, C., et al., "3D bioprinting for engineering complex tissues. Biotechnology advances," 2016. 34(4): p. 422-434.

[15] Nakamura, M., et al. "Computer-Assisted Biofabrication: The challenges on manufacturing 3-D biological tissues for tissue and organ engineering." in VLSI Technology (VLSIT), 2011 Symposium on. 2011. IEEE.

[16] Cui, H., et al., "3D bioprinting for organ regeneration.” Advanced healthcare materials, 2017. 6(1): p. 1601118.

[17] Lee, V.K., et al., "3D bioprinting and 3D imaging for stem cell engineering, in Bioprinting in Regenerative Medicine." 2015, Springer. p. 33-66.

[18] Fermeiro, J., M. Calado, and I. Correia. "State of the art and challenges in bioprinting technologies, contribution of the 3D bioprinting in Tissue Engineering. in Bioengineering (ENBENG), " 2015 IEEE 4th Portuguese Meeting on. 2015. IEEE.

[19] Nakamura, M., et al., "Bioprinting with pre-cultured cellular constructs towards tissue engineering of hierarchical tissues." International Journal of Bioprinting, 2015. 1(1): p. 39-48.

[20] Whitaker, M., "The history of 3D printing in healthcare." The Bulletin of the Royal College of Surgeons of England, 2014. 96(7): p. 228-229.

[21] Boyd, R.L., R. Miller, and V. Vlaskalic, "The Invisalign system in adult orthodontics: mild crowding and space closure cases." Journal of Clinical Orthodontics, 2000. 34(4): p. 203-212.

[22] Ventola, C.L., Medical applications for 3D printing: current and projected uses. Pharmacy and Therapeutics, 2014. 39(10): p. 704.

[23] Lim, K.H.A., et al., "Use of 3D printed models in medical education: A randomized control trial comparing 3D prints versus cadaveric materials for learning external cardiac anatomy." Anatomical sciences education, 2016. 9(3): p. 213-221.

[24] Rengier, F., et al., "3D printing based on imaging data: review of medical applications." International journal of computer assisted radiology and surgery, 2010. 5(4): p. 335-341.

[25] Mahmoud, A. and M. Bennett, "Introducing 3-dimensional printing of a human anatomic pathology specimen: potential benefits for undergraduate and postgraduate education and anatomic pathology practice." Archives of pathology \& laboratory medicine, 2015. 139(8): p. 1048-1051.

[26] Lioufas, P.A., et al., "3D printed models of cleft palate pathology for surgical education." Plastic and Reconstructive Surgery Global Open, 2016; 4(9).

\section{BIOGRAPHIES OF AUTHORS}

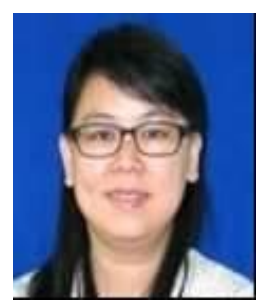

Chin Fhong Soon received the B.Eng. degree (Hons.) in electronic system and medical electronic engineering and Master degree in electronic engineering from Institut Teknologi Tun Hussein Onn in 1998 and 2001 respectively. She received the Ph.D. degree in molecular and biomedical engineering from University Of Bradford, U.K., 2011. She is currently an Associate Professor and the centre head of Biosensor and Bioengineering Laboratory, MiNT-SRC, Universiti Tun Hussein Onn Malaysia. Her current research interests are medical engineering and bio-sensor technology.

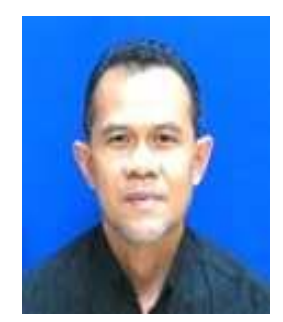

Dirman Hanafi received the B.Eng. degree (Hons.) in electrical engineering from Universitas Bung Hatta, Indonesia, in 1994, and his Master degree in electrical and control engineering from Institut Teknologi Bandung, Indonesia, in 1998, and the Ph.D. degree in electrical engineering from Universiti Teknologi Malaysia, Malaysia, in 2006. He is currently an Associate Professor, as well as a researcher and a lecturer in Faculty of Electrical and Electronic Engineering, Universiti Tun Hussein Onn Malaysia. His current research interests are intelligent control system, robotics and artificial intelligence. 

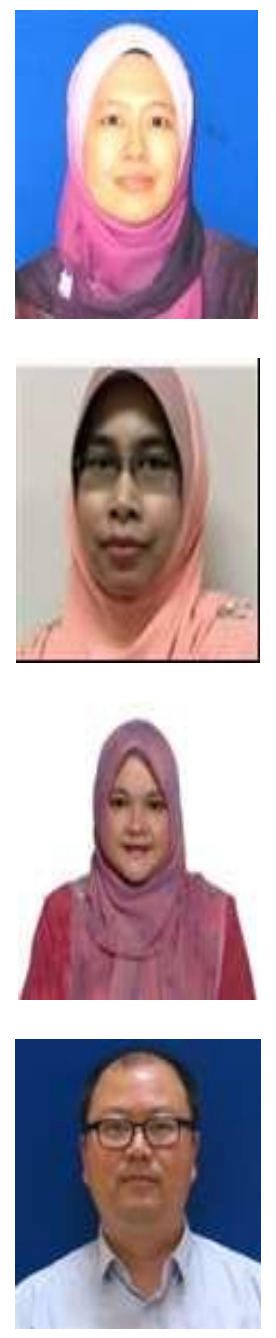

Wan Nurshazwani Binti Wan Zakaria received the B.Eng. degree (Hons.) in mechanical and electronic engineering from Chiba University, Japan in 2007. She received the Master degree in mechatronic engineering and the Ph.D. degree in mechanical engineering from Newcastle University, U.K., in 2008 and 2012 respectively. She is currently the department head of Department of Mechatronics and Robotics Engineering, Faculty of Electrical and Electronic Engineering, Universiti Tun Hussein Onn Malaysia. Her current research interests are mechatronics and robotics.

Safinaz Binti Mohd Khialdin is a paediatric ophthalmologist and currently serves as both a medical lecturer and a consultant ophthalmologist in Department of Ophthalmology, Universiti Kebangsaan Malaysia Medical Centre (UKMMC), Malaysia. Her current research interests are paediatric ophthalmology and vision impairment in children.

Hazlita Isa received the Bachelors of Medicine and Bachelor of Surgery (MBChB) from University of Glasgow, UK, in 1997. She received the Masters in Surgery (Ophthalmology) from Universiti Kebangasaan Malaysia, Malaysia in 2006, and the Ph.D. degree from University College of London, U.K., in 2015. She is currently an ophthalmologist and is currently working in Gleneagles Kuala Lumpur, Malaysia. Her current research interests are cataract, age macula degeneration, diabetic retinopathy, uveitis.

Kian Sek Tee received the B.Eng. degree (Hons.) in electrical engineering from Universiti Teknologi Malaysia, Malaysia in 1997, and the Ph.D. degree in mechatronic engineering from University Of Leeds, U.K., in 2012. He is currently an Associate Professor, as well as a researcher and a lecturer in Faculty of Electrical and Electronic Engineering, Universiti Tun Hussein Onn Malaysia. His current research interests are industrial automation system, mechatronic system in gait monitoring, rehabilitation and exoskeleton. 